\section{Résistance de contact des balais}

Le professeur E. Arnold, et son assistant M. E. Pfiffrer, ont récemment publié, dans l'Elektrotechnische Zeitschrift, les résultats d'expériences très" intéressantes qu'ils ont entreprises au laboratoire de l'Institut électrotechnique de l'Ecole technique supérieure de Karlsruhe, sur la résistance de contact des balais.

Entre un balai et un collecteur, ou une bage, il y a une différence de potentiel $\Delta P$ qui dépend :

a) De la nature du balai et de celle du collecteur ou des bagues;

b) De la densité du courant;

c) Du sens du courant

d) De la température du contact ;

e) De la constitution chimique des surfaces de contact;

f) De la pression;

g) De la vitesse ;

h) De la nature du courant (continu, alternatif ou ondulé).

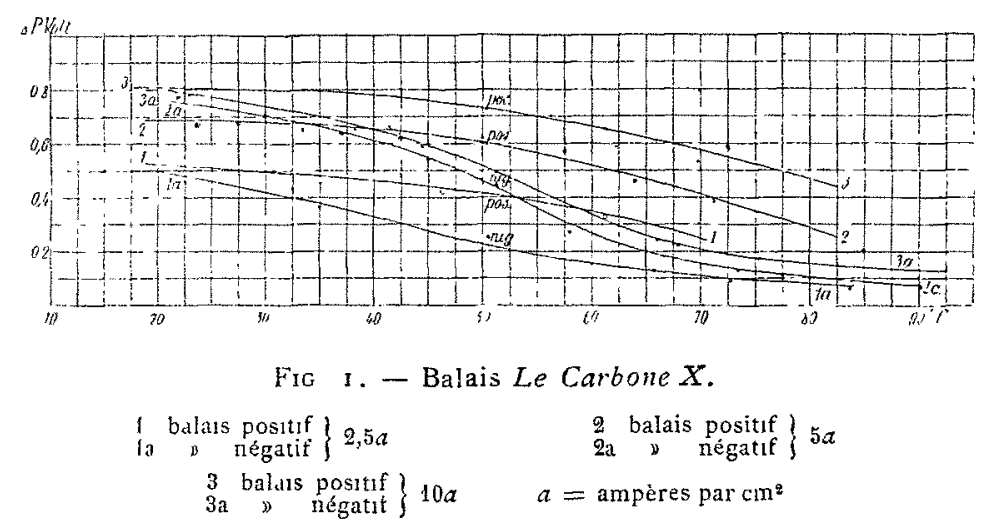

Les essais entrepris jusqu'ici par M. Arnold, et précédemment décrits dans son traité sur la machine à courant continu, étajent rclatifs à la variation de la résistance de contact en fonction de la densité de courant, de la pression, de la vitesse, et de la nature du courant. L'influence de la température n'avait pas été étudiée, car l'obtention de diverses températures, et leur mesure avec les collecteurs, ou les bagues ordinaires, employés lors de ces essais, étaient impossibles.

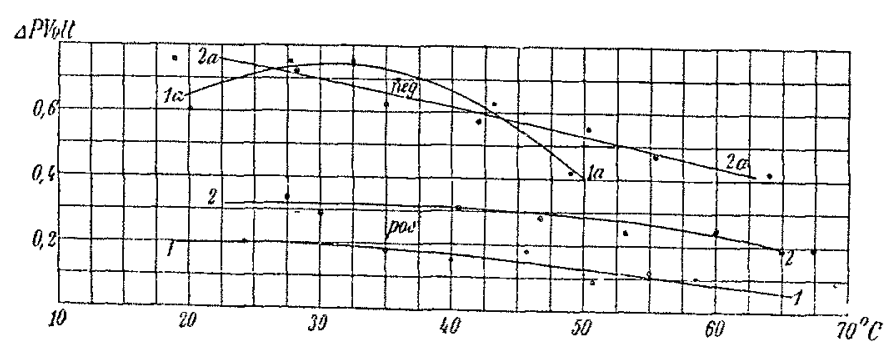

Fig. 2. - Balais Morgante 2 (faibles densités).

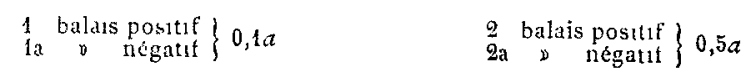

M. Arnold a imaginé un nouveau dispositif : une bague en bronze est montée sur un arbre, et le courant passe par par un balai frottant sur cette bague, puis arrive à une seconde bague, sur laquelle frotte également un balai. La bague en bronze est chauffée au moyen de deux résistances, formées de rubans de nickeline, placées de part et d'autre, au-dessous d'elle. Par l'intermédiaire de deux autres bagues, on peut amener du courant à ces résistances, qui peuvent être disposées en série ou en parallèle. Suivant la valeur de la température ambiante, un courant d'une intensité de i 5 à 18 ampères suffit pour que la bague atteigne une température de 80 à $100^{\circ}$.

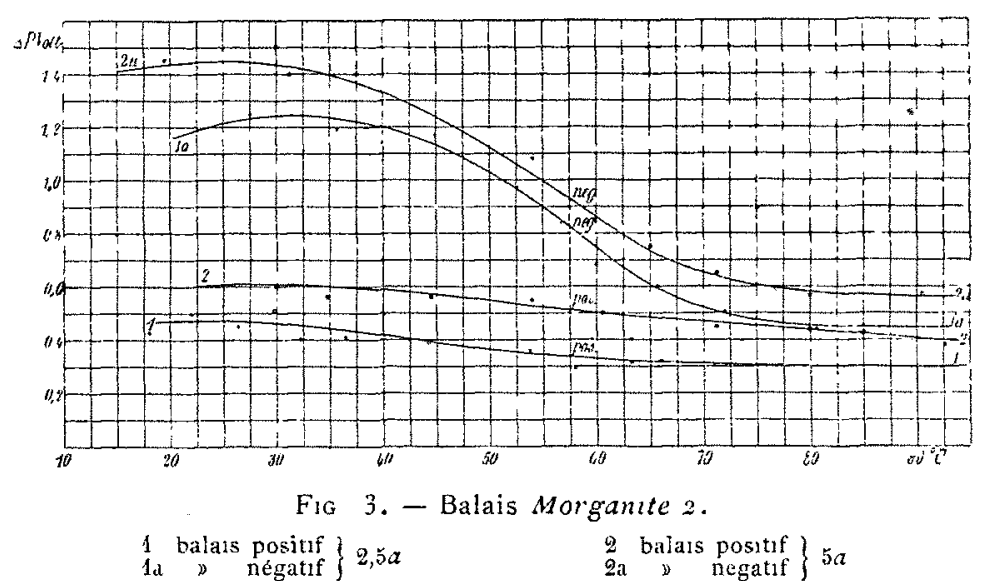

On détermine la température d'après l'augmentation de résistance d'une bobine, d'une résistance de 100 ohms, enroulée contre la bague, de part et d'autre. Les extrémités de cette résistance sont reliées à des conducteurs disposés dans l'axe de l'arbre, et terminés par des pointes plongeant dans du mercure; la résistance de contact est alors absolument négligeable, de sorte que la température peut être déterminée d'après l'augmentation de résistance.

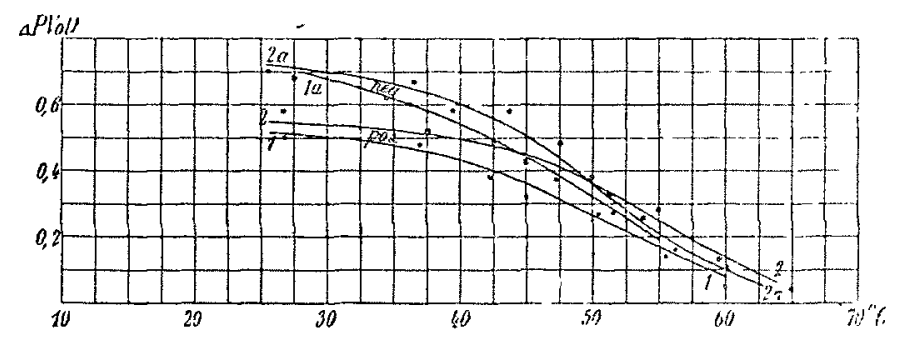

Fig 4. - Balais Le Carbone Z (faibles densités).

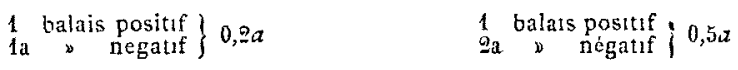

Avant de procéder aux relevés, les expérimentateurs ont fait diverses mesures qui ont prouvé que la bobine prenait très exactement la température de la bague.

Dans tous les essais, la vitesse périphérique de la bague était de $7^{\mathrm{m}} 4$ o par seconde, et la pression de 160 grammes par centimètre carré. Afin d'obtenir des résultats bien exacts, on faisait fonctionner un balai pendant plusieurs jours de suite avant de procéder aux relevés.

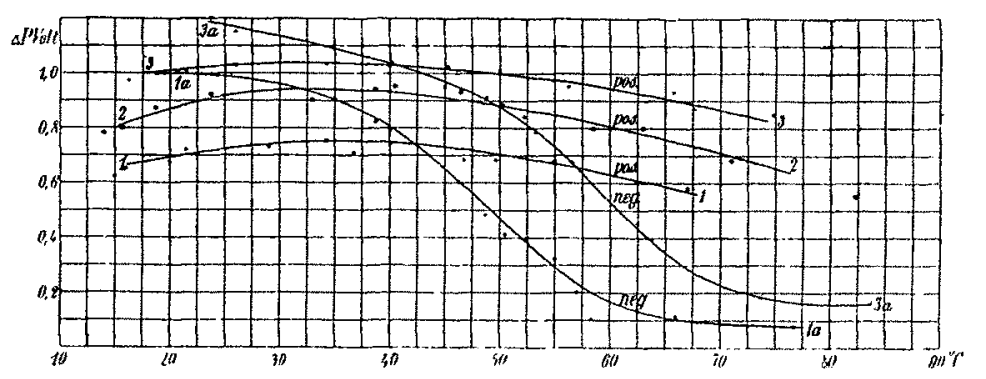

Fig. 5. - Balass Le Carbone Z.

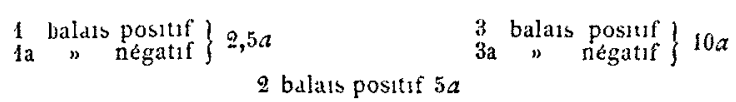

Les essais précédents avaient nettement montré que la résistance de contact varie avec la densité du courant, de sorte que, pour n'avoir comme variable que la température, la densité du courant a été maintenue constante. 
Chacune des courbes relevées par les expérimentateurs est le résultat des essais d'un jour entier. Les relevés ont été exécutés à des intervales de 30 minutes au plus; on n'a pas obtenu des valeurs absolument constantes de la chute de tension, celle-ci oscillant entre un maximum et un minimum, mais on a pris la moyenne.

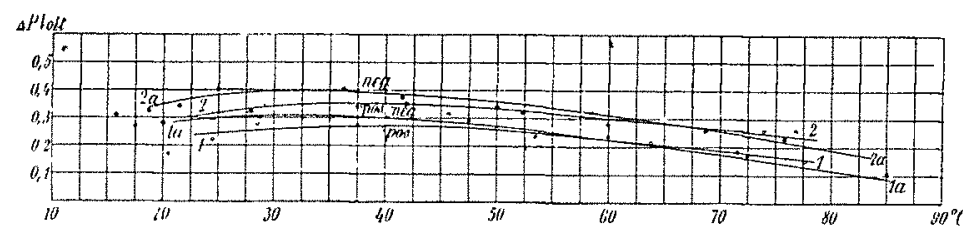

Fig. 6. - Balais 'Endruwett.

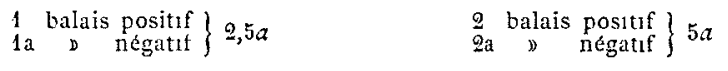

La manière dont se comporte le balai suivant la direction du courant est particulièrement intéressante. Le balai positit (direction du courant de la bague au balai) se comporte avec une plus grande régularité que le balai négatif quand la température augmente. Pour de basses températures, la chute de tension au balai négatif est, en général, plus grande qu'au balai positit, tandis que, pour de hautes températures, c'est le contraire qui a lieu, de sorte qu'il en résulte que les courbes donnant, pour chacun des balais, la chute de tension en fonction de la température, se coupent.

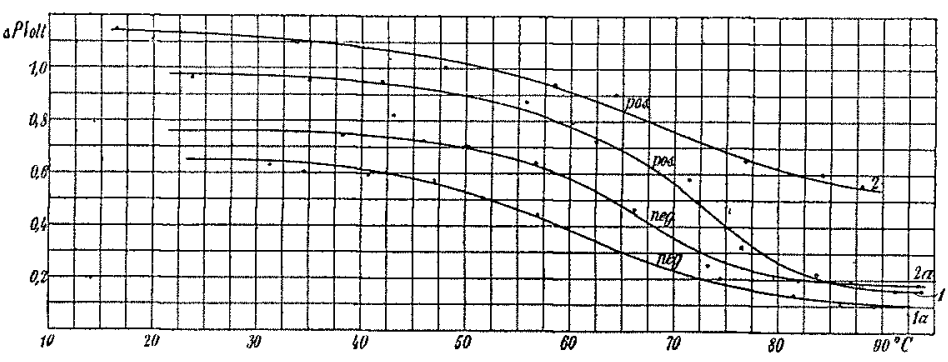

FIG. 7. - Balais Siemens $S$.

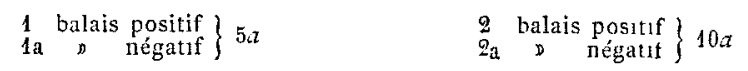

Ceci explique les contradictions des anciens résultats, et le fait que, quand la charge d'une dynamo augmente, ce qui augmente par suite sa température, c'est au balai négatif que commencent d'abord les étincelles.

L'examen des courbes ci-jointes, relevées avec divers balais, montre bien ces faits.

La table suivante donne la résistance de contact, en

\begin{tabular}{|c|c|c|c|c|c|}
\hline \multirow{2}{*}{$\begin{array}{l}\text { QUALITÉ } \\
\text { DU BALAI }\end{array}$} & \multirow{2}{*}{ POLE } & \multicolumn{3}{|c|}{$\begin{array}{l}\text { OIINS } \\
\text { PAR CAI }\end{array}$} & \\
\hline & & 300 & $40^{\circ}$ & $50^{\circ}$ & $60^{\circ}$ \\
\hline $\begin{array}{l}\text { Morganite no } 2 \text {, pour une densité } \\
\text { de } 0,1 \text { ampire pour } \mathrm{cn}^{2} \text {. }\end{array}$ & $\therefore$ & $\begin{array}{l}1,9 \\
7,4\end{array}$ & $\begin{array}{l}1.7 \\
6,7\end{array}$ & $\begin{array}{l}1,3 \\
4,0\end{array}$ & $\begin{array}{c}0,8 \\
n\end{array}$ \\
\hline $\begin{array}{l}\text { Le carbone } 7 \text {, pour une denstéc } \\
\text { de } 0,2 \text { ampere par } \mathrm{cm}^{2} . . .\end{array}$ & -1 & $\begin{array}{l}2,5 \\
5,4\end{array}$ & $\begin{array}{l}2,1 \\
2,7\end{array}$ & $\begin{array}{l}1,9 \\
1,6\end{array}$ & $\begin{array}{c}0,4 \\
0\end{array}$ \\
\hline
\end{tabular}
ohms par $\mathrm{cm}^{2}$ pour de faibles densités de courant.

La table suivante donne la chute de tension en centièmes par rapport à celle correspondant à la température de 350 , pour des densités du courant de 5 amperes par $\mathrm{cm}^{2}$, à l'exception du carbone $Z$, pour lequel l'intensité était de ro am-

\begin{tabular}{|c|c|c|c|c|c|c|c|c|}
\hline \multirow{2}{*}{$\begin{array}{l}\text { QUALI'TE } \\
\text { DU BALAL }\end{array}$} & \multirow{2}{*}{ POLE } & \multicolumn{7}{|c|}{$\begin{array}{l}\text { CHUTE DE TENSION } \\
\text { reLATIVE }\end{array}$} \\
\hline & & 350 & $45^{\circ}$ & $55^{\circ}$ & $65^{\circ}$ & $75^{\circ}$ & 850 & 950 \\
\hline Le Carbone X. & $t$ & $\begin{array}{l}100 \\
100\end{array}$ & $\begin{array}{l}94 \\
85\end{array}$ & $\begin{array}{l}81 \\
53\end{array}$ & $\begin{array}{l}70 \\
30\end{array}$ & $\begin{array}{l}52 \\
21\end{array}$ & $\begin{array}{l}30 \\
15\end{array}$ & 10 \\
\hline Le Carboue Z. & \pm & $\begin{array}{l}100 \\
100\end{array}$ & $\begin{array}{r}100 \\
89\end{array}$ & $\begin{array}{l}9 / 4 \\
67\end{array}$ & $\begin{array}{l}88 \\
32\end{array}$ & $\begin{array}{l}80 \\
16\end{array}$ & $"$ & " \\
\hline Norganite & \pm & $\begin{array}{l}100 \\
100\end{array}$ & $\begin{array}{l}95 \\
88\end{array}$ & $\begin{array}{l}87 \\
72\end{array}$ & $\begin{array}{l}80 \\
51\end{array}$ & $\begin{array}{l}75 \\
43\end{array}$ & $\begin{array}{l}70 \\
38\end{array}$ & $\begin{array}{l}67 \\
37\end{array}$ \\
\hline Balai métallıques & -1 & 100 & 95 & 82 & 62 & $"$ & $"$ & $"$ \\
\hline Endruwert . . & - & 100 & 89 & 54 & 23 & 12 & $"$ & $D$ \\
\hline Balar métal-car- & + & 100 & 97 & 91 & 80 & 69 & $"$ & $"$ \\
\hline bone Endruwellf & - & 100 & 95 & 85 & 70 & 55 & " & $"$ \\
\hline Sicmens $S$ & +- & 100 & 96 & 89 & 32 & 12 & 20 & 19 \\
\hline ' & - & 100 & 89 & $7 \%$ & 47 & 28 & 19 & 15 \\
\hline
\end{tabular}
péres par centimètre carré.
On voit donc que la dimınution de la résistance de contact devient très importante au fur et à mesure de l'augmentation de la température.

Le balai métal-carbone Endrumeit est constitué de minces feuilles de cuivre électrolytique séparées par des couches de carbone, de sorte que la résistance de contact est faible, mais que la résistance pour des courants dirigés perpendiculairement au balai est grande. Le but de cette disposition est de n'obtenir que des pertes très réduites all contact et le long des balais. alors que ceux-ci opposent une résistance très grande aux courants parasites qui se développent dans les bobines en coart circuit et qui se ferment à travers les balais. Les courbes de la figure ô se rapportent à des balais de ce type.

On peut voir sur la figure 7 que le balai positif en charbon Siemens $S$ a, quelle que soit la température, une chute de tension plus élevée que le balai négatuf.

La condition d'une bonne commutation est :

$$
\Delta P>1,25 E_{s}
$$

$E_{s}$ étant la tension efficace due à la réactance d'une bobine de l'induit en court circuit, tension qui oscille ordinairement entre 0,3 et 0,5 volt.

Si la différence de potentiel $\Delta P$ s'abaisse au-dessous de la valeur $1,25 E_{s}$, la commutation risque de devenir mauvaise. Il est bien certain qu'on perd une certaile quantité d'énergie en employant des balais ayant une résistance de contact élerée, mais cet inconvénient est bien inférieur à celui qu'occasionne le crachement des balais. On doit donc employer des balais en charbon présentant une résistance de contact qui soit suffisamment grande et qui se maintienne le plus possible constante.

Il parait donc avantageux, dans le cas de commutation difficile, d'employer des balais en charbon Siemens $S$, ou du Carbone $Z$, du côté positif, et des balais en morganile comme négatifs.

On a fait une deuxième série d'essai pour se rendre compte de la manière dont se comportent les balais à température corstante et densité de courant variable. Ces essais ont été effectués avec les balais morganite et Siemens $S$; les courbes donnant la résistance de contact en fonction de la densité ont l'aspect d'hyperboles presques équilatères. Void 
quelles est la valeur de la résistance de contact en ohms par $\mathrm{cm}^{2}$ pour deux espèces de balkis.

Pour un balai en morganite, à la température de $40^{\circ}$ cette résistance est :

pour le positif :

$$
\begin{aligned}
& R_{c}=\frac{0,35}{d}+0,04 \\
& R_{c}=\frac{1,18}{d}+0,04
\end{aligned}
$$

pour le négatif :

$d$ étant la densité en ampères par $\mathrm{cm}^{2}$.

A $60^{\circ}$, cette résistance devient:

pour le négatif :

$$
\begin{aligned}
& R_{\mathrm{c}}=\frac{0,2}{d}+0,0+ \\
& R_{\mathrm{c}}=\frac{0,6}{d}+0,05
\end{aligned}
$$

pour le positif :

Pour un balai Siemens $S$, on a, à $40^{\circ}$ :

pour le positif :

$$
\begin{aligned}
& R_{c}=\frac{0,84}{d}+0,02 \\
& R_{c}=\frac{0,70}{d}+0,02
\end{aligned}
$$

pour le négatif:

$$
\begin{aligned}
& R_{c}=\frac{0,4}{d}+0,04 \\
& R_{c}=\frac{0,35}{d}+0,02
\end{aligned}
$$

Il est à rebiarquer que la manière dont se compoitent les balais suivant les variations de la température, de la densıté, de la direction du courant, de la vitesse et de la pression ne sont pas absolument uniformes. Dans le cours d'un essai, on a observé parfors des variations brusques de la chute de tension, qui duraient plus ou moins longtemps, et dont on ne ne peut encore donner aucune explication plausible, même par une observation très rigoureuse.

Le but principal des recherches précédentes, qui était de trouver expérimentalement quelle est l'influence de la température du collecteur et des balais sur la chute de tension, a été pleinement atteint. Toutefois, il reste encore un problème à resoudre, c'est celui de la détermination des phéncmènes physiques et chimiques qui se produisent réellement aux surfaces de contact.

\section{G. Picker, \\ Ancien élève de l'Ecole Polytechnique de Karlsruhe.}

\section{Accidents dus au courant électrique en Suisse pendant l'année 1906}

A. Accidents de personnes. - Pour I 9 victimes sur 35, les blessures ont été mortelles (contre 2 I cas mortels dans l'annéc précédente). Les 35 victimes se répartissent de la manière suivante par rapport à leur position vis-à-vis des entreprises électriques respectives.

Personnel d'exploitation proprement dite : 8 (7 en 1905).

Personnel des entreprises étrangères à l'exploitation : 16 (16 en 1905).

Des tiers : ro ( 7 en rgo5).

La plupart des victimes en I 906 se recrutent de nouveau, comme les années précédentes, parmi le personnel des monteurs. Bien que ce fait s'explique en quelque sorte par l'occu. pation même des victimes, il y a pourtant de nouveau à obser- ver qu'une partie des accidents sont dus à l'insouciance du personnel et qu'ils auraient pu être évités si toutes les instructions avaient été strictement observées, et si l'on avait pris les mesures de précaution s'imposant à tout homme du mètier plus ou moins instruit. Le personnel d'exploitation figure également assez souvent dans les accidents dus à la propre faute de la victime. C'est bien dans cette categorie qu'il faut mentionner les ro accidents qui ont eu lieu lors des travaux dans des stations de transformation ou de distribution à haute tension non déclanchées, et un accident sur une ligne à haute tension également non déclanchée.

Dans un cas, un monteur avait travaillé le samedi et le dimanche dans une station de transformation qu'on avait déclanchée du réseau en enlevant les connexions sur la tourelle de distribution. Ayant terminé son travail plus tôt qu'il n'avait pensé, il voulut refaire les ligatures sur la tourelle, dans la supposition que la ligne n'était pas sous tension le dimanche, et malgré que ce n'était pas son devoir et qu'il savait qu'un autre monteur était chargé de ce travail. La conduite était sous courant et le monteur fut tué aussitôt qu'il toucha les fils.

Dans un autre cas, un monteur voulut commencer de vernir les ferrures dans une station de transformation, avant l'heure qui avait été fixée pour l'interruption du courant. En glissant, il toucha les conduitcs à baute tension avec sa figure, se qui occasionna sa mort.

Dans un troisième cas, un gardien d'une station transformatrice était occupé à enlever des toiles d'araignées entre les conduites à haute tension. Il entra en contact avec un des fils et fut foudroyé.

On ne saurait donc assez répéter la nécessité de rendre le personnel d'exploitation et les monteurs toujours très attentifs au danger et d'exiger de leur part toules les mesures de précaulion. If faudra surtout interdire rigoureusement tout travail aux installations à haute tension sous courant, et insister pour qu'on metre en court-circuit et à la terre les installations à haute tension, toutes les fois que les circonstances le permettent, avant d'y entreprendre des travaux.

Il est important de prendre toutes les mesures de précaution, lors même que tout danger pour les ouvriers parait exclu.

Le cas suivant en démontre la nécessité.

Un monteur avait travaillé entre midi et une heure dans une station de trazsformation qu'il avait déclanché lui-même. A une heure un aide-monteur enclancha par erreur la station malgré un ordre contraire qui lui avait été donné. Le monteur qui n'avait pas encore terminé son travail, fut foudroyé.

Le nombre des accidents où des personnes étrangères à l'entreprise ont été victimes a augmenté et les 44 pour roo de ces cas concernent des ouvriers du bâtiment.

Fn partie, ces accidents peuvent être attribués à un avertissement insulfisant sur le danger des conduites électriques; mais, d'autre part, il arrive assez fréquemment que les ouvriers du bâtiment ne tiennent pas compte de ces avertissements, comme le prouve le cas suivant.

Un menuisier travaillant sur un échafa ıdage touciıa un fil d'une conduite à basse tension (à 220 volts), pour prouver à ses camarades que cela pouvait se faire sans danger. Ensuite de la commotion qu'il ressentit, il risqua de tomber de l'échafaudage, et saisit instinctivement l'autre fil de la conduite électrique. II fut tué par le courant qui traversa son corps. Lesconduites étaient protégées par des planches contre un contactaccidentel.

Le cas suivant doit, par contre, être attribué à un avertissement insuffisant .

Un manœuvre d'un entrepreneur de bâtiments avait reçu l'ordre d'encaisser une conduite à basse tension sous courant, pour empêcher un contact accidentel des fils nus. Pendant son travail, il toucha la conduite et se brûla à la main droite, on ne l'avait pas averti qu'll y avait danger de toucher aux fils. 